\title{
On the Mode Theory of Very-Low-Frequency Propagation in the Presence of a Transverse Magnetic Field

\author{
D. D. Crombie ${ }^{1}$
}

(December 14, 1959)

\begin{abstract}
The effect of a purely transverse horizontal magnetic field on the propagation of verylow-frequency (vlf) waves is considered. It is shown that the magnetic field introduces nonreciprocity, and that for propagation along the magnetic equator, the rate of attenuation is less for west-to-east propagation than for east-to-west propagation.
\end{abstract}

\section{Introduction}

It has been shown by Budden [1] $]^{2}$ and by Wait [2] that the condition for vlf waves to be guided between a plane, perfectly conducting earth, and a plane, uniform, sharply bounded ionosphere is,

$$
R=\exp \left(4 \pi j h C_{n} / \lambda-2 \pi j n\right)
$$

where

$n=$ order of mode,

$h / \lambda=$ height of ionosphere in wavelengths,

$R=$ the reflection coefficient of the ionosphere, and

$C_{n}=$ the cosine of the complex angle of incidence $\theta_{n}$ of the wave on the ionosphere, for the $n$th mode.

It can be shown that the vertical component of the electric field of a given mode, $n$, at the ground in the earth-ionosphere waveguide is proportional to

$$
\exp j\left(\omega t-2 \pi d S_{n} / \lambda\right)
$$

where $S_{n}$ is the sine of the angle of incidence. If the wave is attenuated in the guide $\theta_{n}, C_{n}$, and $S_{n}$ are complex. A mode travels with a phase velocity

$$
v_{p}=c / \operatorname{Re} S_{n}
$$

and suffers an attenuation

$$
A=k d \operatorname{Im} S_{n} \text {, nepers/unit distance. }
$$

In these expressions, $c$ is the velocity of light, $d$ is the horizontal distance traveled, Re $S_{n}$ and $\mathrm{Im} \cdot S_{n}$ are the real and imaginary parts of $S_{n}$, and $k$ is $2 \pi / \lambda$.

Budden [1] and Wait [2] have discussed the behavior of $v_{p}$ and $A$ for cases in which the earth's magnetic field can be neglected. Their treatment requires the substitution of (1) of the appropriate Fresnel reflection coefficient for a sharply bounded ionosphere, followed by the solution of (1) for $C_{n}$.

Barber and Crombie [3] have recently shown that if the ionosphere is assumed to be sharply bounded and the earth's magnetic field is taken to be purely transverse, then the reflection coefficient for waves polarized in the plane of incidence is given by the complex quantity

1 Dominion Physical Laboratory, Department of Scientific and Industrial Research, Lower Hutt, New Zealand (invited paper).

2 Figures in brackets indicate the literature references at the end of this paper. 


$$
R=\frac{C_{n}\left[(1+\delta)^{2}-\gamma^{2}\right]-\left[\mathrm{cm}_{2}\left(\delta+\delta^{2}-\gamma^{2}\right) / \omega-j \gamma S_{n}\right]}{C_{n}\left[(1+\delta)^{2}-\gamma^{2}\right]+\left[\mathrm{cm}_{2}\left(\delta+\delta^{2}-\gamma^{2}\right) / \omega-j \gamma S_{n}\right]}
$$

At vlf $\delta \cong j \beta=j \omega \nu / \omega_{0}^{2}, \gamma=\omega \cdot \omega_{H} / \omega_{0}^{2}$, where $\omega / 2 \pi$ is the wave frequency, $\omega_{0} / 2 \pi$ is the critical frequency of the ionosphere, and $\omega_{H} / 2 \pi$ is the gyrofrequency of the electrons in the ionosphere. The term $\mathrm{cm}_{2} / \omega$ is given by

$$
\mathrm{cm}_{2} / \omega=\sqrt{C_{n}^{2}+(1+j \beta) /\left(j \beta-\beta^{2}-\gamma^{2}\right)}
$$

the sign in front of the radical being chosen so that the imaginary part of $\mathrm{cm}_{2} / \omega$ is negative. This ensures that the amplitude of the wave transmitted into the ionosphere decreases with height.

For propagation from west to east, the sign of $\gamma$ is negative, while for east-to-west propagation it is positive. Thus the value of $R$, in general, differs for these two directions of propagation, and the earth-ionosphere waveguide exhibits nonreciprocal propagation as will be demonstrated below.

\section{Propagation Constant of the Earth-Ionosphere Waveguide}

Signals received over very great distances must suffer little attenuation; thus $|R| \cong 1$. Then, eq (5) can be written as

$$
\left.\begin{array}{rl}
R & =-(1-x) \\
\text { where } x & =\frac{2 C_{n}\left[(1+j \beta)^{2}-\gamma^{2}\right]}{\operatorname{cm}_{2}\left(j \beta-\beta^{2}-\gamma^{2}\right) / \omega-j \gamma S_{n}}
\end{array}\right\} .
$$

From eq (1), taking natural logarithms

$$
C_{n}=\frac{(n-1 / 2)}{2 h / \lambda}+\frac{j x}{2 k h},
$$

$x$ is a function of $C_{n}$ and $S_{n}$. Following Wait [2], a first-order perturbation solution of (7) can be obtained if $C_{n}$ and $S_{n}$ in $x$ are replaced by $\bar{C}_{n}=\frac{(n-1 / 2)}{2 h / \lambda}+$ and $\bar{S}_{n}=\sqrt{1-\bar{C}_{n}^{2}}$, the values obtained from (7) when $x=0$.

Then

$$
S_{n}=\bar{S}_{n}-\frac{j x \bar{C}_{n}}{2 k h \bar{S}_{n}}
$$

From (5a) and (6), since $\bar{C}_{n}^{2}$ is very small for low order modes,

$$
x=-2 \frac{\bar{C}_{n}\left[1-\beta^{2}-\gamma^{2}+2 j \beta\right] \sqrt{j \beta}}{j \beta(1+j \beta) \sqrt{1-\gamma^{2} / j \beta(1+j \beta)}+j \gamma \bar{S}_{n} \sqrt{j \beta}} .
$$

It is assumed that $\gamma^{2}<|\beta(1+j \beta)|$, and on taking $\sqrt{j}$ as $-(1+j) / \sqrt{2}$

$$
x=\frac{2 \bar{C}_{n} \sqrt{2 \beta}\left(1-\beta^{2}-\gamma^{2}+2 j \beta\right)(1+j)}{-2 \beta^{2}-\gamma^{2}+\gamma \bar{S}_{n} \sqrt{2 \beta}+j\left(2 \beta-\sqrt{2 \beta} \gamma \bar{S}_{n}\right.} .
$$

Separating this into its real and imaginary parts and substituting these in (8) gives

$$
\begin{aligned}
\operatorname{Im} S_{n} & =-\frac{\bar{C}_{n}^{2} \sqrt{2 \beta}}{k h \bar{S}_{n}} \cdot \frac{2 \beta\left(1+\beta+\beta^{2}+\beta^{3}\right)+\gamma^{2}\left(3 \beta^{2}+\gamma^{2}-1\right)-4 \beta \sqrt{2 \beta} \gamma \bar{S}_{n}}{4 \beta \gamma^{2} \bar{S}_{n}^{2}+\left(2 \beta^{2}+\gamma^{2}\right)^{2}+4 \beta^{2}-2 \gamma \bar{S}_{n} \sqrt{2 \beta}\left(2 \beta^{2}+2 \beta+\gamma^{2}\right)} \\
\operatorname{Re} S_{n} & =\bar{S}_{n}+\frac{\bar{C}_{n}^{2} \sqrt{2 \beta}}{k h \bar{S}_{n}} \cdot \frac{2 \gamma \bar{S}_{n} \sqrt{2 \beta}\left(1-\beta^{2}-\gamma^{2}\right)-2 \beta\left(1-\beta+\beta^{2}-\beta^{3}\right)-\gamma^{2}\left(1-3 \beta^{2}-\gamma^{2}\right)}{4 \beta \gamma^{2} \bar{S}_{n}^{2}+\left(2 \beta^{2}+\gamma^{2}\right)^{2}+4 \beta^{2}-2 \gamma \bar{S}_{n} \sqrt{2 \beta}\left(2 \beta^{2}+2 \beta+\gamma^{2}\right)} .
\end{aligned}
$$


It is instructive to simplify these equations further by letting $\gamma$ be much less than $\beta$. Equation (9) then reduces to

$$
\operatorname{Im} S_{n}=-\frac{\bar{C}_{n}^{2}}{2 \sqrt{2} k h \bar{S}_{n}}\left(\frac{1}{\sqrt{\beta}}+\sqrt{\beta}\right)\left[1+\frac{\gamma \bar{S}_{n} \sqrt{2}}{(1+\beta) \sqrt{\beta}}\right] .
$$

This is identical, when $\gamma=0$, to the value obtained by Wait [2] for propagation in the absence of the earth's magnetic field. Equation (11) shows that for west-to-east propagation, since $\gamma$ is then negative, the attenuation coefficient is less than when the field is absent $(\gamma=0)$ or when the propagation is from east to west ( $\gamma$ positive).

From eq (11) the ratio of the west-to-east, and east-to-west attenuation coefficients can be written as

$$
\frac{\operatorname{Im} S_{n}(W \rightarrow E)}{\operatorname{Im} S_{n}(E \rightarrow W)} \simeq 1-\frac{2 \sqrt{2} \gamma \bar{S}_{n}}{(1+\beta) \sqrt{\beta}} \simeq 1-\frac{2 \sqrt{2} \omega_{H} \omega_{n}}{\nu^{3 / 2} \omega^{1 / 2}}
$$

when $\beta$ is greater than unity. The value of this ratio decreases as the wavelength increases, and thus the nonreciprocity becomes more marked at the lower frequencies.

When $\gamma \ll \beta$, eq $(10)$ reduces to

$$
\operatorname{Re} S_{n} \simeq \bar{S}_{n}+\frac{\bar{C}_{n}^{2}}{2 \sqrt{2} k h \bar{S}_{n}}\left(\sqrt{\beta}-\frac{1}{\sqrt{\beta}}\right)
$$

which again is the value obtained by Wait in the absence of a magnetic field. Thus the presence of a small transverse magnetic field does not affect the phase velocity of waves in the earth ionosphere waveguide under the conditions assumed here.

When the transverse magnetic field is such that $\gamma$ and $\beta$ are comparable, eqs (9) and (10) must be considered in full. Some calculations for likely ionospheric parameters have shown that nonreciprocity is still present under this condition.

\section{Discussion}

The writer has shown previously [4] that there is a considerable amount of evidence indicating that long nighttime vlf paths show nonreciprocal effects. The data refer to paths which cut the magnetic equator at various angles and thus the results cannot be really compared with the present work which deals only with a purely transverse horizontal, and small, magnetic field. It was not possible from the experimental evidence mentioned above to determine whether daytime paths exhibited reciprocity since in most cases the signals received over the daytime paths were too small.

However, when the ionosphere is dark reflection takes place from a greater height than when it is daylit, and thus the collision frequency $\nu$ is reduced considerably. Equation (11) shows that the effect of this is to make the nonreciprocity larger. Thus it may be expected that the effect of the transverse component of the earth's magnetic field will be smaller for a daytime path than for a nighttime path.

\section{References}

[1] K. G. Budden, The propagation of a radio atmospheric, Phil. Mag. 42, 1 (1951)

[2] J. R. Wait, The mode theory of VLF ionospheric propagation for finite ground conductivity, Proc. IRE 45, 760 (1957). See also J. R. Wait, J. Research NBS 64D, 153 (1960) for a discussion of the anisotropic case.

[3] N. F. Barber and D. D. Crombie, VLF reflections from the ionosphere in the presence of a transveres magnetic field (to be published in J. Atmospheric and Terrest. Phys.).

[4] D. D. Crombie, Differences between the east-west and west-east propagation of VLF signals over long distances, J. Atmospheric and Terrest. Phys. 12, 110 (1958). 